\title{
Extended Auxiliary Equation Method and Its Applications to Three Generalized NLS Equations
}

\author{
Gui-qiong Xu \\ Department of Information Management, College of Management, Shanghai University, Shanghai 200444, China \\ Correspondence should be addressed to Gui-qiong Xu; xugq@staff.shu.edu.cn
}

Received 7 January 2014; Revised 19 February 2014; Accepted 20 February 2014; Published 31 March 2014

Academic Editor: Baojian Hong

Copyright (C) 2014 Gui-qiong Xu. This is an open access article distributed under the Creative Commons Attribution License, which permits unrestricted use, distribution, and reproduction in any medium, provided the original work is properly cited.

The auxiliary equation method proposed by Sirendaoreji is extended to construct new types of elliptic function solutions of nonlinear evolution equations. The effectiveness of the extended method is demonstrated by applications to the RKL model, the generalized derivative NLS equation and the Kundu-Eckhaus equation. Not only are the Jacobian elliptic function solutions are derived, but also the solitary wave solutions and trigonometric function solutions are obtained in a unified way.

\section{Introduction}

Partial differential equations describe various nonlinear phenomena in natural and applied sciences such as fluid dynamics, plasma physics, solid state physics, optical fibers, acoustics, biology, and mathematical finance. It is of significant importance to solve nonlinear partial differential equations (NLPDEs) from both theoretical and practical points of view. In the past decades, many powerful methods for solving NLPDEs have been developed, such as the inverse scattering method [1], Bäcklund and Darboux transform [2, 3], Hirota's bilinear method [4], Painlevé analysis method [5], variable separation method [6-8], tanh-function method [911], variational iteration method [12], homotopy perturbation method [13], Jacobian elliptic function expansion [14-20], F-expansion method [21,22], and Fan subequation method $[23,24]$ and its various extensions [25-29].

Sirendaoreji $[30,31]$ presented a new auxiliary equation method by introducing a new first-order nonlinear ordinary differential equation:

$$
\left(\frac{\mathrm{d} \phi}{\mathrm{d} \xi}\right)^{2}=c_{2} \phi^{2}(\xi)+c_{4} \phi^{4}(\xi)+c_{6} \phi^{6}(\xi), \quad \phi=\phi(\xi),
$$

where $c_{j}(j=2,4,6)$ are real parameters to be determined. The key idea of the auxiliary equation method is to use the solutions of (1) instead of $\tanh \xi$ in tanh-function method and extended tanh-function method [23]. Zhang and his coworker [32], Huang et al. [33], and Yomba [34] have improved the Sirendaoreji method in different manners.

The structure of this paper is organized as follows. In Section 2, an extended auxiliary equation method is described to construct exact solutions of NLPDEs. In Section 3, we apply this improved method to three generalized NLS equations with cubic-quintic terms. Some conclusions are given in Section 4.

\section{An Extended Auxiliary Equation Method}

For the sake of simplicity, we assume JacobiSN $(\xi, m)=\operatorname{sn}(\xi)$, $\operatorname{JacobiCN}(\xi, m)=\mathrm{cn}(\xi)$, and $\operatorname{JacobiDN}(\xi, m)=\operatorname{dn}(\xi)$. The derivatives of the above three kinds of Jacobian elliptic functions satisfy [35]:

$$
\begin{gathered}
\operatorname{sn}^{\prime}(\xi)=\operatorname{cn}(\xi) \operatorname{dn}(\xi), \quad \operatorname{cn}^{\prime}(\xi)=-\operatorname{sn}(\xi) \mathrm{dn}(\xi), \\
\operatorname{dn}^{\prime}(\xi)=-m^{2} \operatorname{sn}(\xi) \operatorname{cn}(\xi),
\end{gathered}
$$

where $^{\prime}=\mathrm{d} / \mathrm{d} \xi$, and $m$ is the modulus of Jacobian elliptic functions $(0 \leq m \leq 1)$.

For a given NLPDE, with independent variables $(x, t)$ and dependent variable $u$.

Step 1. Use the travelling wave transformation $u(x, t)=u(\xi)$, $\xi=k(x-\omega t)$, and reduce the given NLPDE:

$$
H\left(u, u_{x}, u_{t}, u_{x x}, u_{x t}, \ldots\right)=0,
$$


to the following ordinary differential equation

$$
G\left(u, u^{\prime}, u^{\prime \prime}, \ldots\right)=0,
$$

where' denotes $\mathrm{d} / \mathrm{d} \xi$.

Step 2. The solutions of (4) can be supposed as

$$
u(\xi)=F\left(\phi^{i}(\xi)\right)
$$

where $F$ is a suitable variable transformation, and $\phi(\xi)$ satisfies the following first-order differential equation:

$$
\left(\frac{\mathrm{d} \phi}{\mathrm{d} \xi}\right)^{2}=c_{0}+c_{2} \phi^{2}(\xi)+c_{4} \phi^{4}(\xi)+c_{6} \phi^{6}(\xi),
$$

which is slightly different from (1) by adding one constant term $c_{0}$. The crucial step is to give the solutions of (6). It is difficult to give the general solution of (6); here we only consider twelve solutions expressed by various kinds of Jacobian elliptic functions, which read

$$
\phi(\xi)=\frac{1}{2}\left[-\frac{c_{4}}{c_{6}}(1 \pm f(\xi))\right]^{1 / 2},
$$

where the function $f(\xi)$ could be expressed through elliptic functions $s n, c n, d n$, their inverse and different ratios like $\mathrm{sn} / \mathrm{cn}, \mathrm{dn} / \mathrm{sn}$ and so on.

Type I. If $c_{0}=c_{4}^{3}\left(m^{2}-1\right) /\left(32 c_{6}^{2} m^{2}\right), c_{2}=c_{4}^{2}\left(5 m^{2}-1\right) /\left(16 c_{6} m^{2}\right)$, and $c_{6}>0, f(\xi)$ in (7) takes the form

$$
f_{1}(\xi)=\operatorname{sn}(\rho \xi), \quad f_{2}(\xi)=\frac{1}{m \operatorname{sn}(\rho \xi)}, \quad \rho=\frac{c_{4} \sqrt{c_{6}}}{2 m c_{6}} .
$$

Type II. If $c_{0}=c_{4}^{3}\left(1-m^{2}\right) /\left(32 c_{6}^{2}\right), c_{2}=c_{4}^{2}\left(5-m^{2}\right) /\left(16 c_{6}\right)$, and $c_{6}>0, f(\xi)$ in $(7)$ takes the form

$$
f_{3}(\xi)=m \operatorname{sn}(\rho \xi), \quad f_{4}(\xi)=\frac{1}{\operatorname{sn}(\rho \xi)}, \quad \rho=\frac{c_{4} \sqrt{c_{6}}}{2 c_{6}} .
$$

Type III. If $c_{0}=c_{4}^{3} /\left(32 m^{2} c_{6}^{2}\right), c_{2}=c_{4}^{2}\left(4 m^{2}+1\right) /\left(16 c_{6} m^{2}\right)$, and $c_{6}<0, f(\xi)$ in (7) takes the form

$$
\begin{gathered}
f_{5}(\xi)=\operatorname{cn}(\rho \xi), \quad f_{6}(\xi)=\frac{\sqrt{1-m^{2}} \operatorname{sn}(\rho \xi)}{\operatorname{dn}(\rho \xi)}, \\
\rho=\frac{c_{4} \sqrt{-c_{6}}}{2 m c_{6}} .
\end{gathered}
$$

Type IV. If $c_{0}=c_{4}^{3} m^{2} /\left(32 c_{6}^{2}\left(m^{2}-1\right)\right), c_{2}=c_{4}^{2}\left(5 m^{2}-\right.$ $4) /\left(16 c_{6}\left(m^{2}-1\right)\right)$, and $c_{6}<0, f(\xi)$ in (7) takes the form

$$
\begin{gathered}
f_{7}(\xi)=\frac{\sqrt{1-m^{2}} \operatorname{dn}(\rho \xi)}{1-m^{2}}, \quad f_{8}(\xi)=\frac{1}{\operatorname{dn}(\rho \xi)}, \\
\rho=\frac{c_{4} \sqrt{c_{6}\left(m^{2}-1\right)}}{2 c_{6}\left(m^{2}-1\right)} .
\end{gathered}
$$

Type $V$. If $c_{0}=c_{4}^{3} /\left(32 c_{6}^{2}\left(1-m^{2}\right)\right), c_{2}=c_{4}^{2}\left(4 m^{2}-5\right) /\left(16 c_{6}\left(m^{2}-\right.\right.$ $1))$, and $c_{6}>0, f(\xi)$ in (7) takes the form

$$
\begin{gathered}
f_{9}(\xi)=\frac{1}{\operatorname{cn}(\rho \xi)}, \quad f_{10}(\xi)=\frac{\sqrt{1-m^{2}} \mathrm{dn}(\rho \xi)}{\left(1-m^{2}\right) \operatorname{sn}(\rho \xi)}, \\
\rho=\frac{c_{4} \sqrt{c_{6}\left(1-m^{2}\right)}}{2 c_{6}\left(1-m^{2}\right)} .
\end{gathered}
$$

Type VI. If $c_{0}=m^{2} c_{4}^{3} /\left(32 c_{6}\right), c_{2}=c_{4}^{2}\left(m^{2}+4\right) /\left(16 c_{6}\right)$, and $c_{6}<0, f(\xi)$ in $(7)$ takes the form

$$
\begin{gathered}
f_{11}(\xi)=\operatorname{dn}(\rho \xi), \quad f_{12}=\frac{\sqrt{1-m^{2}}}{\operatorname{dn}(\rho \xi)}, \\
\rho=\frac{c_{4} \sqrt{-c_{6}}}{2 c_{6}} .
\end{gathered}
$$

Step 3. Substituting (7) together with (8)-(13) into (5), some new types of Jacobian elliptic function solutions of (3) can be obtained in a unified way.

With the aid of the computer algebraic software Maple, the solutions given by (7)-(13) have been verified by putting them back to the original equation (6). To our knowledge, these twelve solutions are firstly reported here. When the modulus $m$ approaches 1 or 0 , the Jacobian elliptic functions degenerate to hyperbolic functions and trigonometric functions, respectively.

\section{Applications}

In this section, three generalized NLS equations with physical interests are chosen to illustrate the effectiveness of the above method.

3.1. The RKL Model. Let us first consider the third-order generalized NLS equation [36], which is proposed by Radhakrishnan, Kundu, and Lakshmanan (RKL). The normalized RKL model can be written as

$$
\begin{aligned}
i u_{z}+ & u_{t t}+2|u|^{2} u+i \alpha u_{t t t}+i \beta\left(|u|^{2} u\right)_{t} \\
& +i \gamma\left(|u|^{4} u\right)_{t}+\delta|u|^{4} u=0
\end{aligned}
$$

which describes the propagation of femtosecond optical pulses. In (14), $u=u(z, t)$ represents a normalized complex slowly varying amplitude of the pulse envelope, and $\alpha, \beta, \gamma, \delta$ are real constants. Some solitary wave solutions and combined Jacobian elliptic function solution were constructed by different methods [37-39].

In order to solve (14), its solutions may be supposed as

$$
\begin{gathered}
u(z, t)=\phi(\xi) \mathrm{e}^{i \eta}, \quad \xi=k t+\omega z, \\
\eta=\lambda t+\mu z,
\end{gathered}
$$


where $k, \omega, \lambda$, and $\mu$ are real constants. Substituting (15) into (14) and taking real and imaginary parts separately, we have

$$
\begin{aligned}
& (3 \alpha \lambda-1) k^{2} \phi^{\prime \prime}(\xi) \\
& =\left(\alpha \lambda^{3}-\lambda^{2}-\mu\right) \phi(\xi)+(2-\lambda \beta) \phi^{3}(\xi) \\
& \quad+(\delta-\lambda \gamma) \phi^{5}(\xi), \\
& \alpha k^{3} \phi^{\prime \prime \prime}(\xi)+\left(\omega+2 \lambda k-3 \alpha \lambda^{2} k\right) \phi^{\prime}(\xi) \\
& \quad+3 \beta k \phi^{2}(\xi) \phi^{\prime}(\xi)+5 \gamma k \phi^{4}(\xi) \phi^{\prime}(\xi)=0 .
\end{aligned}
$$

There are two cases to discuss.

Case 1. When $3 \alpha \lambda-1 \neq 0$.

Integrating (17) and setting the integration constant to zero, we obtain

$$
\alpha k^{3} \phi^{\prime \prime}(\xi)=-\left(\omega+2 \lambda k-3 \alpha \lambda^{2} k\right) \phi(\xi)-\beta k \phi^{3}(\xi)-\gamma k \phi^{5}(\xi) .
$$

Equations (16) and (18) will be equivalent, provided that

$$
\frac{(3 \alpha \lambda-1) k^{2}}{\alpha k^{3}}=\frac{\alpha \lambda^{3}-\lambda^{2}-\mu}{-\left(\omega+2 \lambda k-3 \alpha \lambda^{2} k\right)}=\frac{2-\lambda \beta}{-\beta k}=\frac{\delta-\lambda \gamma}{-\gamma k} .
$$

From which we get

$$
\begin{aligned}
& \lambda=\frac{\beta-2 \alpha}{2 \alpha \beta}, \quad \gamma=\frac{\beta \delta}{2}, \\
& \omega=\frac{2 k \alpha\left(4 \beta-8 \alpha+\mu \beta^{3}\right)}{\beta^{2}(\beta-6 \alpha)} .
\end{aligned}
$$

Under the parametric constraints (20), integrating (18) and setting the integration constant to zero, we have

$$
\phi^{\prime 2}(\xi)=2 A+c_{2} \phi^{2}(\xi)+c_{4} \phi^{4}(\xi)+c_{6} \phi(\xi)^{6},
$$

where $A$ is an arbitrary integration constant, and $c_{2}, c_{4}, c_{6}$ are given by

$$
\begin{gathered}
c_{2}=\frac{8 \alpha^{3}+8 \alpha^{2} \mu \beta^{3}+\beta^{3}-2 \alpha \beta^{2}-4 \beta \alpha^{2}}{4 k^{2} \alpha^{2} \beta^{2}(6 \alpha-\beta)}, \\
c_{4}=-\frac{\beta}{2 \alpha k^{2}}, \quad c_{6}=-\frac{\beta \delta}{6 \alpha k^{2}} .
\end{gathered}
$$

Up to now, we can obtain twelve Jacobi elliptic function solutions of (14), which can be expressed as the unified form:

$$
\begin{array}{r}
u_{j}(z, t)=\frac{1}{2}\left[-\frac{c_{4}}{c_{6}}\left(1 \pm f_{j}(\xi)\right)\right]^{1 / 2} \mathrm{e}^{i(\lambda t+\mu z)}, \\
\xi=k t+\omega z, \quad j=1, \ldots, 12,
\end{array}
$$

where $\lambda, \gamma, \omega$ are given by (19), $c_{2}, c_{4}$, and $c_{6}$ are given by (22), and $f_{j}(\xi) \quad(j=1, \ldots, 12)$ are given by $(8)-(13)$.
Some solitary wave solutions can be obtained if the modulus $m$ approaches 1 . The solution $u_{1}(z, t)$ given by (23) degenerates to the kink-type solitary wave solution:

$$
\begin{aligned}
u_{13}(z, t) & \\
= & \left\{-\frac{3}{4 \delta}\left[1 \pm \tanh \left(\frac{\sqrt{-6 \alpha \beta \delta}}{4 k \alpha \delta}(k t+\omega z)\right)\right]\right\}^{1 / 2} \\
& \times \mathrm{e}^{i(\lambda t+\mu z)},
\end{aligned}
$$

where $\alpha \beta \delta<0$, and the parameter $\mu$ is determined by $c_{2}=$ $-3 \beta /\left(8 \alpha \delta k^{2}\right)$.

When $m \rightarrow 1$, the solution $u_{5}(z, t)$ given by (23) degenerates to the bell-type solitary wave solution:

$$
\begin{aligned}
u_{14}(z, t) & \\
= & \left\{-\frac{3}{4 \delta}\left[1 \pm \operatorname{sech}\left(\frac{\sqrt{6 \alpha \beta \delta}}{4 k \alpha \delta}(k t+\omega z)\right)\right]\right\}^{1 / 2} \\
& \times \mathrm{e}^{i(\lambda t+\mu z)},
\end{aligned}
$$

where $\alpha \beta \delta>0$, and $\mu$ is determined by $c_{2}=-15 \beta /\left(32 \alpha \delta k^{2}\right)$.

When $m \rightarrow 1$, the solution $u_{2}(z, t)$ degenerates to

$$
\begin{aligned}
u_{15}(z, t) & \\
= & \left\{-\frac{3}{4 \delta}\left[1 \pm \operatorname{coth}\left(\frac{\sqrt{-6 \alpha \beta \delta}}{4 k \alpha \delta}(k t+\omega z)\right)\right]\right\}^{1 / 2} \\
& \times \mathrm{e}^{i(\lambda t+\mu z)},
\end{aligned}
$$

where $\alpha \beta \delta<0$, and $\mu$ is determined by $c_{2}=-3 \beta /\left(8 \alpha \delta k^{2}\right)$.

Some trigonometric function solutions can be obtained if the modulus $m \rightarrow 0$; for example, the solution $u_{4}(z, t)$ becomes

$$
\begin{aligned}
u_{16}(z, t) & \\
= & \left\{-\frac{3}{4 \delta}\left[1 \pm \csc \left(\frac{\sqrt{-6 \alpha \beta \delta}}{4 k \alpha \delta}(k t+\omega z)\right)\right]\right\}^{1 / 2} \\
& \times \mathrm{e}^{i(\lambda t+\mu z)},
\end{aligned}
$$

where $\alpha \beta \delta<0$, and $\mu$ is determined by $c_{2}=-15 \beta /\left(32 \alpha \delta k^{2}\right)$.

When $m \rightarrow 0$, the solution $u_{9}(z, t)$ becomes

$$
\begin{aligned}
u_{17}(z, t) & \\
= & \left\{-\frac{3}{4 \delta}\left[1 \pm \sec \left(\frac{\sqrt{-6 \alpha \beta \delta}}{4 k \alpha \delta}(k t+\omega z)\right)\right]\right\}^{1 / 2} \\
& \times \mathrm{e}^{i(\lambda t+\mu z)},
\end{aligned}
$$

where $\alpha \beta \delta<0$, and $\mu$ is determined by $c_{2}=-15 \beta /\left(32 \alpha \delta k^{2}\right)$.

Case 2. When $3 \alpha \lambda-1=0$, we have

$$
\lambda=\frac{1}{3 \alpha}
$$


then setting the coefficients of $\phi, \phi^{3}$, and $\phi^{5}$ in (16) to zero, respectively, yields

$$
\mu=-\frac{2}{27 \alpha^{2}}, \quad \beta=6 \alpha, \quad \gamma=3 \alpha \delta .
$$

Substituting (29) and (30) into (17) and integrating it with respect to $\xi$, we have

$$
\phi^{\prime 2}(\xi)=2 A+c_{2} \phi^{2}(\xi)+c_{4} \phi^{4}(\xi)+c_{6} \phi^{6}(\xi),
$$

where $A$ is an arbitrary integration constant, and $c_{2}, c_{4}, c_{6}$ are given by

$$
c_{2}=-\frac{3 \alpha \omega+k}{3 \alpha^{2} k^{3}}, \quad c_{4}=-\frac{3}{k^{2}}, \quad c_{6}=-\frac{\delta}{k^{2}} .
$$

Similar to Case 1, we can also get twelve elliptic function solutions of (14), which read

$$
\begin{array}{r}
u_{j}(z, t)=\frac{1}{2}\left[-\frac{c_{4}}{c_{6}}\left(1 \pm f_{j}(\xi)\right)\right]^{1 / 2} \mathrm{e}^{i(\lambda t+\mu z)}, \\
\xi=k t+\omega z, \quad j=18, \ldots, 29,
\end{array}
$$

where the parameters $\lambda, \mu, \beta, \gamma$ are given by (29)-(30), $c_{2}, c_{4}$, and $c_{6}$ are given by $(32)$, and $f_{j}(\xi)(j=18, \ldots, 29)$ are given by $(8)-(13)$.

When the modulus $m \rightarrow 1$, the solution $u_{18}(z, t)$ given by (33) degenerates to the solitary wave solution:

$$
\begin{aligned}
u_{30}(z, t) & \\
= & \frac{1}{2}\left\{-\frac{3}{\delta}\left[1 \pm \tanh \left(\frac{3 \sqrt{-\delta}}{2 k \delta}(k t+\omega z)\right)\right]\right\}^{1 / 2} \\
& \times \mathrm{e}^{i(\lambda t+\mu z)},
\end{aligned}
$$

where $\delta<0$, and $\omega=k\left(27 \alpha^{2}-4 \delta\right) /(12 \alpha \delta)$.

When $m \rightarrow 1$, the solution $u_{19}(z, t)$ degenerates to

$$
\begin{aligned}
u_{31}(z, t) & \\
= & \frac{1}{2}\left\{-\frac{3}{\delta}\left[1 \pm \operatorname{coth}\left(\frac{3 \sqrt{-\delta}}{2 k \delta}(k t+\omega z)\right)\right]\right\}^{1 / 2} \\
& \times \mathrm{e}^{i(\lambda t+\mu z)},
\end{aligned}
$$

where $\delta<0$, and $\omega=k\left(27 \alpha^{2}-4 \delta\right) /(12 \alpha \delta)$.

When $m \rightarrow 1$, the solution $u_{20}(z, t)$ degenerates to the solitary wave solution:

$$
\begin{aligned}
u_{32}(z, t) & \\
= & \frac{1}{2}\left\{-\frac{3}{\delta}\left[1 \pm \operatorname{sech}\left(\frac{3 \sqrt{\delta}}{2 k \delta}(k t+\omega z)\right)\right]\right\}^{1 / 2} \\
& \times \mathrm{e}^{i(\lambda t+\mu z)},
\end{aligned}
$$

where $\delta>0$, and $\omega=k\left(135 \alpha^{2}-16 \delta\right) /(48 \alpha \delta)$.

All solutions given above have been checked with Maple by putting them back into the original equation (14). Among them, only the solutions $u_{13}(z, t), u_{14}(z, t), u_{30}(z, t)$, and $u_{32}(z, t)$ had been found in [38]. To the author's knowledge, the other solutions have not been found before.
3.2. The Generalized Derivative NLS Equation. Next we consider the generalized derivative NLS equation:

$$
i u_{t}+b_{1} u_{x x}+\left(b_{3}|u|^{2}+b_{5}|u|^{4}\right) u-i s_{0} u_{x}-i s_{2}\left(|u|^{2} u\right)_{x}=0
$$

where $b_{1}, b_{3}, b_{5}, s_{0}, s_{2}$ are real constants. Both pulse and fronts that existed in perturbed system (37) were studied by van Saarloos and Hohenberg [40]. Some solitary wave solutions of (37) were obtained by Huang et al. through a generalized auxiliary expansion method [33]. However, the Jacobian elliptic function solutions of (37) have not been reported in literature.

The solutions of (37) may be supposed as

$$
u(x, t)=\phi(\xi) \mathrm{e}^{i(\psi(\xi)-\omega t)}, \quad \xi=k x-\lambda t,
$$

where $k$ and $\lambda$ are constants to be determined. Substituting (38) into (37) and then separating the real and imaginary parts yield the ordinary differential equations about $\phi(\xi)$ and $\psi(\xi)$ :

$$
\begin{aligned}
& 2 b_{1} k^{2} \phi^{\prime}(\xi) \psi^{\prime}(\xi)+b_{1} k^{2} \phi(\xi) \psi^{\prime \prime}(\xi) \\
& -3 k s_{2} \phi^{2}(\xi) \phi^{\prime}(\xi)-\left(k s_{0}+\lambda\right) \phi^{\prime}(\xi)=0, \\
& b_{1} k^{2} \phi^{\prime \prime}(\xi)+\left(\lambda+k s_{0}\right) \phi(\xi) \psi^{\prime}(\xi) \\
& \quad-b_{1} k^{2} \phi(\xi) \psi^{\prime 2}(\xi)+k s_{2} \phi^{3}(\xi) \psi^{\prime}(\xi) \\
& +\omega \phi(\xi)+b_{3} \phi^{3}(\xi)+b_{5} \phi^{5}(\xi)=0 .
\end{aligned}
$$

Under the constraint

$$
\psi^{\prime}(\xi)=\frac{\lambda+k s_{0}}{2 b_{1} k^{2}}+\frac{3 s_{2}}{4 k b_{1}} \phi^{2}(\xi)
$$

equation (39) is satisfied identically, and (40) becomes

$$
\begin{aligned}
& b_{1} k^{2} \phi^{\prime \prime}(\xi) \\
& +\frac{\lambda^{2}+2 \lambda s_{0} k+4 \omega k^{2} b_{1}+s_{0}^{2} k^{2}}{4 k^{2} b_{1}} \phi(\xi) \\
& +\frac{\lambda s_{2}+s_{2} s_{0} k+2 b_{3} k b_{1}}{2 k b_{1}} \phi^{3}(\xi) \\
& +\frac{3 s_{2}^{2}+16 b_{5} b_{1}}{16 b_{1}} \phi^{5}(\xi)=0 .
\end{aligned}
$$

Multiplying (42) by $\phi^{\prime}(\xi)$ and integrating it with respect to $\xi$, we have

$$
\phi^{\prime 2}(\xi)=2 A+c_{2} \phi^{2}(\xi)+c_{4} \phi^{4}(\xi)+c_{6} \phi^{6}(\xi),
$$


where $A$ is an arbitrary integration constant, and $c_{2}, c_{4}, c_{6}$ are given by

$$
\begin{aligned}
& c_{2}=-\frac{\lambda^{2}+2 \lambda s_{0} k+4 \omega k^{2} b_{1}+s_{0}^{2} k^{2}}{4 b_{1}^{2} k^{4}}, \\
& c_{4}=-\frac{\lambda s_{2}+s_{2} s_{0} k+2 b_{3} k b_{1}}{4 b_{1}^{2} k^{3}}, \\
& c_{6}=-\frac{3 s_{2}^{2}+16 b_{5} b_{1}}{48 b_{1}^{2} k^{2}} .
\end{aligned}
$$

Up to now, we can obtain abundant Jacobi elliptic function solutions of (37), which can be expressed as the unified form:

$$
\begin{array}{r}
u_{j}(x, t)=\frac{1}{2}\left[-\frac{c_{4}}{c_{6}}\left(1 \pm f_{j}(\xi)\right)\right]^{1 / 2} \mathrm{e}^{i(\psi(\xi)-\omega t)}, \\
\xi=k x-\lambda t, \quad j=1, \ldots, 12,
\end{array}
$$

where $c_{2}, c_{4}$, and $c_{6}$ are given by $(44), \psi(\xi)$ satisfies the constraint given by (41), and $f_{j}(\xi)$ are given by (8)-(13).

When $m \rightarrow 1$, the solution $u_{1}(x, t)$ given by (45) degenerates to the kink-type solitary wave solution:

$$
\begin{gathered}
u_{13}(x, t)=\phi_{1}(\xi) \mathrm{e}^{i(\psi(\xi)-\omega t)}, \\
\phi_{1}(\xi)=\frac{1}{2}\left\{-\frac{c_{4}}{c_{6}}\left[1 \pm \tanh \left(\frac{c_{4} \sqrt{c_{6}}}{2 m c_{6}} \xi\right)\right]\right\}^{1 / 2}, \\
\psi^{\prime}(\xi)=\frac{\lambda+k s_{0}}{2 b_{1} k^{2}}+\frac{3 s_{2}}{4 k b_{1}} \phi_{1}^{2}(\xi), \quad \xi=k x-\lambda t,
\end{gathered}
$$

where $\omega$ is determined by $c_{2}=c_{4}^{2} /\left(4 c_{6}\right)$.

When $m \rightarrow 1$, the solution $u_{5}(x, t)$ degenerates to the bell-type solitary wave solution:

$$
\begin{gathered}
u_{14}(x, t)=\phi_{2}(\xi) \mathrm{e}^{i(\psi(\xi)-\omega t)}, \\
\phi_{2}(\xi)=\frac{1}{2}\left\{-\frac{c_{4}}{c_{6}}\left[1 \pm \operatorname{sech}\left(\frac{c_{4} \sqrt{-c_{6}}}{2 c_{6}} \xi\right)\right]\right\}^{1 / 2}, \\
\psi^{\prime}(\xi)=\frac{\lambda+k s_{0}}{2 b_{1} k^{2}}+\frac{3 s_{2}}{4 k b_{1}} \phi_{2}^{2}(\xi), \quad \xi=k x-\lambda t,
\end{gathered}
$$

where $\omega$ is determined by $c_{2}=5 c_{4}^{2} /\left(16 c_{6}\right)$. To the authors' knowledge, these fourteen solutions of (37) are firstly reported here.

3.3. The Kundu-Eckhaus Equation. Finally we consider the Kundu-Eckhaus equation with important physical interests:

$$
i u_{t}+u_{x x}+\beta|u|^{2} u+4 \delta^{2}|u|^{4} u+i \delta^{2}\left(|u|^{2}\right)_{x} u=0,
$$

which was derived by Kundu [41, 42] and Eckhaus [43-45] independently and then known as Kundu-Eckhaus equation. In (48), the parameters $\beta, \delta$ are real constants. To the author's knowledge, the integrable nonlinear equation (48) has not been investigated for possible exact solutions through elliptic function.

The solutions of (48) may be supposed as

$$
u(x, t)=\phi(\xi) e^{i(\psi(\xi)-\omega t)}, \quad \xi=k x-\lambda t,
$$

where $k$ and $\lambda$ are constants to be determined. Substituting (49) into (48) and then separating the real and imaginary parts yield the ordinary differential equations about $\phi(\xi)$ and $\psi(\xi)$ :

$$
\begin{aligned}
& -\lambda \phi^{\prime}(\xi)+2 k^{2} \phi^{\prime}(\xi) \psi^{\prime}(\xi)+k^{2} \phi(\xi) \psi^{\prime \prime}(\xi) \\
& \quad+2 k \delta^{2} \phi^{2}(\xi) \phi^{\prime}(\xi)=0, \\
& \lambda \phi(\xi) \psi^{\prime}(\xi)+\omega \phi(\xi)+k^{2} \phi^{\prime \prime}(\xi)-k^{2} \phi(\xi) \psi^{\prime 2}(\xi) \\
& \quad+\beta \phi^{3}(\xi)+4 \delta^{2} \phi^{5}(\xi)=0 .
\end{aligned}
$$

Under the constraint

$$
\psi^{\prime}(\xi)=\frac{\lambda}{2 k^{2}}-\frac{\delta^{2}}{2 k} \phi^{2}(\xi),
$$

equation (50) is satisfied identically, and then multiplying (51) by $\phi^{\prime}(\xi)$ and integrating it with respect to $\xi$, we have

$$
\phi^{\prime 2}(\xi)=2 A+c_{2} \phi^{2}(\xi)+c_{4} \phi^{4}(\xi)+c_{6} \phi^{6}(\xi),
$$

where $A$ is an arbitrary integration constant, and $c_{2}, c_{4}, c_{6}$ are given by

$$
c_{2}=-\frac{\lambda^{2}+4 \omega k^{2}}{4 k^{4}}, \quad c_{4}=-\frac{\beta}{2 k^{2}}, \quad c_{6}=\frac{\delta^{4}-16 \delta^{2}}{12 k^{2}} .
$$

Up to now, we can obtain twelve Jacobi elliptic function solutions of (48), which can be expressed as the unified form:

$$
\begin{array}{r}
u_{j}(x, t)=\frac{1}{2}\left[-\frac{c_{4}}{c_{6}}\left(1 \pm f_{j}(\xi)\right)\right]^{1 / 2} \mathrm{e}^{i(\psi(\xi)-\omega t)}, \\
\xi=k x-\lambda t, \quad j=1, \ldots, 12,
\end{array}
$$

where $c_{2}, c_{4}$, and $c_{6}$ are given by $(54), \psi(\xi)$ satisfies the constraint (52), and $f_{j}(\xi)$ are given by (8)-(13). It is obvious that the solutions of (48) are similar as those of (37) in the form. However, the values of $c_{2}, c_{4}, c_{6}$ as well as the relation between $\psi(\xi)$ and $\phi(\xi)$ are different.

When the modulus $m \rightarrow 1$, we can obtain the solitary wave solutions from (55). If $m \rightarrow 0$, the trigonometric function solutions can be also constructed. For the sake of simplicity, these solutions are not listed here.

\section{Conclusions}

With the aid of symbolic computation software Maple, we present abundant families of new periodic wave solutions for the auxiliary equation (6). Based on these new periodic wave solutions, the auxiliary equation method proposed by Sirendaoreji has been improved and applied to three 
generalized NLS equations with cubic-quintic terms. As a result, a series of new travelling wave solutions have been obtained including not only Jacobian elliptic function solutions but also solitary wave solutions and trigonometric function solutions. These solutions may be important to explain some physical phenomena and find applications in the nonlinear pulse propagation through optical fibers.

The improved auxiliary equation method can be used for solving other nonlinear partial differential equations in mathematical physics, for example, the generalized PochhammerChree equation [46]:

$$
u_{t t}-u_{t t x x}-\left(a_{1} u+a_{3} u^{3}+a_{5} u^{5}\right)_{x x}=0,
$$

the $(2+1)$-dimensional cubic-quintic Ginzburg-Landau equation [47]:

$$
\begin{aligned}
i u_{z} & +\frac{1}{2} u_{x x}+\frac{1}{2}(\beta-i) u_{\tau \tau}+i u+\left(1-i r_{1}\right)|u|^{2} u \\
& +i r_{2}|u|^{4} u=0
\end{aligned}
$$

the generalized long-short wave resonance equation [48]:

$$
\begin{gathered}
i S_{t}+S_{x x}=\alpha L S+\gamma|S|^{2} S+\delta|S|^{4} S, \\
L_{t}+\beta|S|_{x}^{2}=0,
\end{gathered}
$$

and so on. How to construct other types of exact solutions for nonlinear models with cubic-quintic nonlinear terms? It is an interesting and significant topic, and this problem is still under investigation.

\section{Conflict of Interests}

The author declares that there is no conflict of interests regarding the publication of this paper.

\section{Acknowledgments}

The author is greatly indebted to the reviewer for his (or her) helpful comments and constructive suggestions. This work was supported by the Natural Science Foundation of China under Grant no. 11201290.

\section{References}

[1] M. J. Ablowitz and P. A. Clarkson, Solitons, Nonlinear Evolution Equations and Inverse Scattering, Cambridge University Press, Cambridge, UK, 1991.

[2] C. Rogers and W. F. Shadwick, Bäcklund Transformations and Their Applications, vol. 161 of Mathematics in Science and Engineering, Academic Press, New York, NY, USA, 1982.

[3] V. B. Matveev and M. A. Salle, Darboux Transformations and Solitons, Springer Series in Nonlinear Dynamics, Springer, Berlin, Germany, 1991.

[4] R. Hirota, The Direct Method in Soliton Theory, vol. 155 of Cambridge Tracts in Mathematics, Cambridge University Press, Cambridge, UK, 2004.
[5] J. Weiss, M. Tabor, and G. Carnevale, "The Painlevé property for partial differential equations," Journal of Mathematical Physics, vol. 24 , no. 3, pp. 522-526, 1983.

[6] S.-Y. Lou, "On the coherent structures of the Nizhnik-NovikovVeselov equation," Physics Letters A, vol. 277, no. 2, pp. 94-100, 2000.

[7] R. Radha, C. S. Kumar, M. Lakshmanan, and C. R. Gilson, "The collision of multimode dromions and a firewall in the two-component long-wave-short-wave resonance interaction equation," Journal of Physics A, vol. 42, no. 10, Article ID 102002, 2009.

[8] G.-Q. Xu and X.-Z. Huang, "New variable separation solutions for two nonlinear evolution equations in higher dimensions," Chinese Physics Letters, vol. 30, no. 3, Article ID 130202, 2013.

[9] W. Malfliet, "Solitary wave solutions of nonlinear wave equations," American Journal of Physics, vol. 60, no. 7, pp. 650-654, 1992.

[10] R.-X. Yao and Z.-B. Li, "New exact solutions for three nonlinear evolution equations," Physics Letters A, vol. 297, no. 3-4, pp. 196204, 2002.

[11] Y.-P. Liu and Z.-B. Li, "A Maple package for finding exact solitary wave solutions of coupled nonlinear evolution equations," Computer Physics Communications, vol. 155, no. 1, pp. 65-76, 2003.

[12] M. A. Abdou and A. A. Soliman, "New applications of variational iteration method," Physica D, vol. 211, no. 1-2, pp. 1-8, 2005.

[13] J.-H. He, "Application of homotopy perturbation method to nonlinear wave equations," Chaos, Solitons and Fractals, vol. 26, no. 3, pp. 695-700, 2005.

[14] S. Liu, Z. Fu, S. Liu, and Q. Zhao, "Jacobi elliptic function expansion method and periodic wave solutions of nonlinear wave equations," Physics Letters A, vol. 289, no. 1-2, pp. 69-74, 2001.

[15] G.-Q. Xu and Z.-B. Li, "On the Painlevé integrability, periodic wave solutions and soliton solutions of generalized coupled higher-order nonlinear Schrödinger equations," Chaos, Solitons and Fractals, vol. 26, no. 5, pp. 1363-1375, 2005.

[16] G.-Q. Xu and Z.-B. Li, "Applications of Jacobi elliptic function expansion method for nonlinear differential-difference equations," Communications in Theoretical Physics, vol. 43, no. 3, pp. 385-388, 2005.

[17] G.-Q. Xu, "New types of exact solutions for the fourth-order dispersive cubic-quintic nonlinear Schrödinger equation," Applied Mathematics and Computation, vol. 217, no. 12, pp. 5967-5971, 2011.

[18] Z. Yan, "Periodic, solitary and rational wave solutions of the 3D extended quantum Zakharov-Kuznetsov equation in dense quantum plasmas," Physics Letters A, vol. 373, no. 29, pp. 24322437, 2009.

[19] B. Hong, "New exact Jacobi elliptic functions solutions for the generalized coupled Hirota-Satsuma KdV system," Applied Mathematics and Computation, vol. 217, no. 2, pp. 472-479, 2010.

[20] B. Hong and D. Lu, "New Jacobi elliptic function-like solutions for the general KdV equation with variable coefficients," Mathematical and Computer Modelling, vol. 55, no. 3-4, pp. 1594-1600, 2012.

[21] Y. Zhou, M. Wang, and Y. Wang, "Periodic wave solutions to a coupled KdV equations with variable coefficients," Physics Letters A, vol. 308, no. 1, pp. 31-36, 2003. 
[22] E. Yomba, "The extended F-expansion method and its application for solving the nonlinear wave, CKGZ, GDS, DS and GZ equations," Physics Letters A, vol. 340, no. 1-4, pp. 149-160, 2005.

[23] E. Fan, "Extended tanh-function method and its applications to nonlinear equations," Physics Letters A, vol. 277, no. 4-5, pp. 212-218, 2000.

[24] E. Fan, "Multiple travelling wave solutions of nonlinear evolution equations using a unified algebraic method," Journal of Physics A, vol. 35, no. 32, pp. 6853-6872, 2002.

[25] G. Xu, "An elliptic equation method and its applications in nonlinear evolution equations," Chaos, Solitons and Fractals, vol. 29, no. 4, pp. 942-947, 2006.

[26] Y. Chen and Z. Yan, "Weierstrass semi-rational expansion method and new doubly periodic solutions of the generalized Hirota-Satsuma coupled KdV system," Applied Mathematics and Computation, vol. 177, no. 1, pp. 85-91, 2006.

[27] D.-S. Wang and H.-B. Li, “Elliptic equation's new solutions and their applications to two nonlinear partial differential equations," Applied Mathematics and Computation, vol. 188, no. 1, pp. 762-771, 2007.

[28] O. P. Layeni, "A new rational auxiliary equation method and exact solutions of a generalized Zakharov system," Applied Mathematics and Computation, vol. 215, no. 8, pp. 2901-2907, 2009.

[29] H. Li, K. Wang, and J. Li, "Exact traveling wave solutions for the Benjamin-Bona-Mahony equation by improved Fan subequation method," Applied Mathematical Modelling, vol. 37, no. 14-15, pp. 7644-7652, 2013.

[30] S. Sirendaoreji, "A new auxiliary equation and exact travelling wave solutions of nonlinear equations," Physics Letters A, vol. 356, no. 2, pp. 124-130, 2006.

[31] S. Sirendaoreji, "Auxiliary equation method and new solutions of Klein-Gordon equations," Chaos, Solitons and Fractals, vol. 31, no. 4, pp. 943-950, 2007.

[32] S. Zhang and T.-C. Xia, "A generalized auxiliary equation method and its application to $(2+1)$-dimensional asymmetric Nizhnik-Novikov-Vesselov equations," Journal of Physics A, vol. 40, no. 2, pp. 227-248, 2007.

[33] D.-J. Huang, D.-S. Li, and H.-Q. Zhang, "Explicit and exact travelling wave solutions for the generalized derivative Schrödinger equation," Chaos, Solitons and Fractals, vol. 31, no. 3, pp. 586593, 2007.

[34] E. Yomba, “The sub-ODE method for finding exact travelling wave solutions of generalized nonlinear Camassa-Holm, and generalized nonlinear Schrödinger equations," Physics Letters A, vol. 372, no. 3, pp. 215-222, 2008.

[35] M. Abramowitz and I. Stegun, Handbook of Mathematical Functions with Formulas, Graphs, and Mathematical Tables, Dover, New York, NY, USA, 1992.

[36] R. Radhakrishnan, A. Kundu, and M. Lakshmanan, "Coupled nonlinear Schrödinger equations with cubic-quintic nonlinearity: integrability and soliton interaction in non-Kerr media," Physical Review E, vol. 60, no. 3, pp. 3314-3323, 1999.

[37] W.-P. Hong, "Optical solitary wave solutions for the higher order nonlinear Schrödinger equation with cubic-quintic nonKerr terms," Optics Communications, vol. 194, no. 1-3, pp. 217223, 2001.

[38] M. Wang, X. Li, and J. Zhang, "Sub-ODE method and solitary wave solutions for higher order nonlinear Schrödinger equation," Physics Letters A, vol. 363, no. 1-2, pp. 96-101, 2007.
[39] H. Triki and T. R. Taha, "Exact analytic solitary wave solutions for the RKL model," Mathematics and Computers in Simulation, vol. 80, no. 4, pp. 849-854, 2009.

[40] W. van Saarloos and P. C. Hohenberg, "Fronts, pulses, sources and sinks in generalized complex Ginzburg-Landau equations," Physica D, vol. 56, no. 4, pp. 303-367, 1992.

[41] A. Kundu, "Landau-Lifshitz and higher-order nonlinear systems gauge generated from nonlinear Schrödinger type equations," Journal of Mathematical Physics, vol. 25, no. 12, pp. 34333438, 1984.

[42] A. Kundu, "Exact solutions to higher-order nonlinear equations through gauge transformation," Physica D, vol. 25, no. 1-3, pp. 399-406, 1987.

[43] W. Eckhaus, The Long-Time Behaviour forPerturbed WaveEquations and Related Problems, Preprint no. 404, Department of Mathematics, University of Utrecht, 1985.

[44] W. Eckhaus, "The long-time behaviour for perturbed waveequations and related problems," in Trends in Applications of Pure Mathematics to Mechanics, vol. 249 of Lecture Notes in Physics, pp. 168-194, Springer, Berlin, Germany, 1986.

[45] F. Calogero and W. Eckhaus, "Nonlinear evolution equations, rescalings, model PDEs and their integrability. I," Inverse Problems, vol. 3, no. 2, pp. 229-262, 1987.

[46] W.-G. Zhang and W.-X. Ma, "Explicit solitary-wave solutions to generalized Pochhammer-Chree equations," Applied Mathematics and Mechanics, vol. 20, no. 6, pp. 625-632, 1999.

[47] H. Sakaguchi and B. A. Malomed, "Stable localized pulses and zigzag stripes in a two-dimensional diffractive-diffusive Ginzburg-Landau equation," Physica D, vol. 159, no. 1-2, pp. 91100, 2001.

[48] Y. Shang, "Explicit and exact solutions for a generalized longshort wave resonance equations with strong nonlinear term," Chaos, Solitons and Fractals, vol. 26, no. 2, pp. 527-539, 2005. 


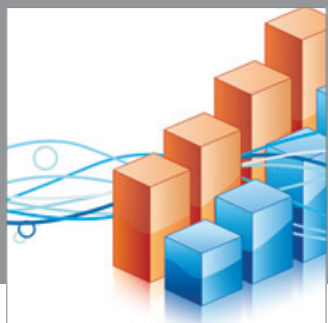

Advances in

Operations Research

mansans

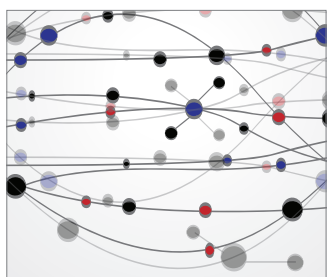

The Scientific World Journal
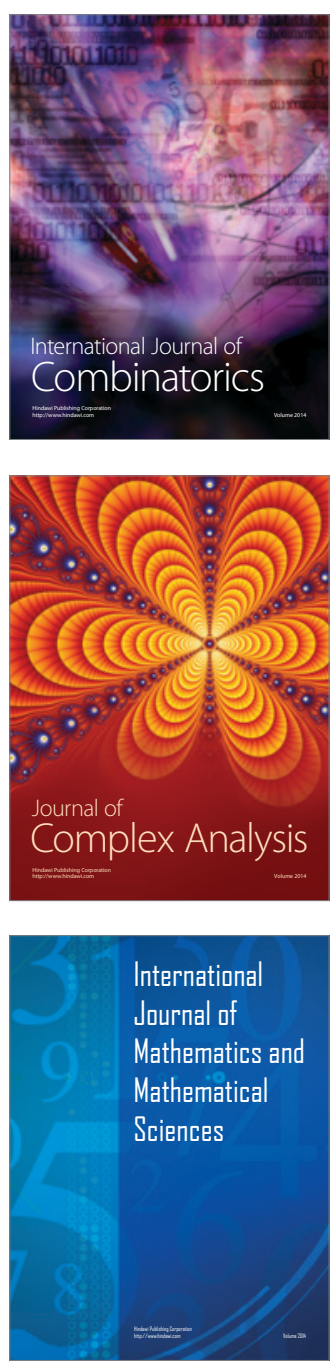
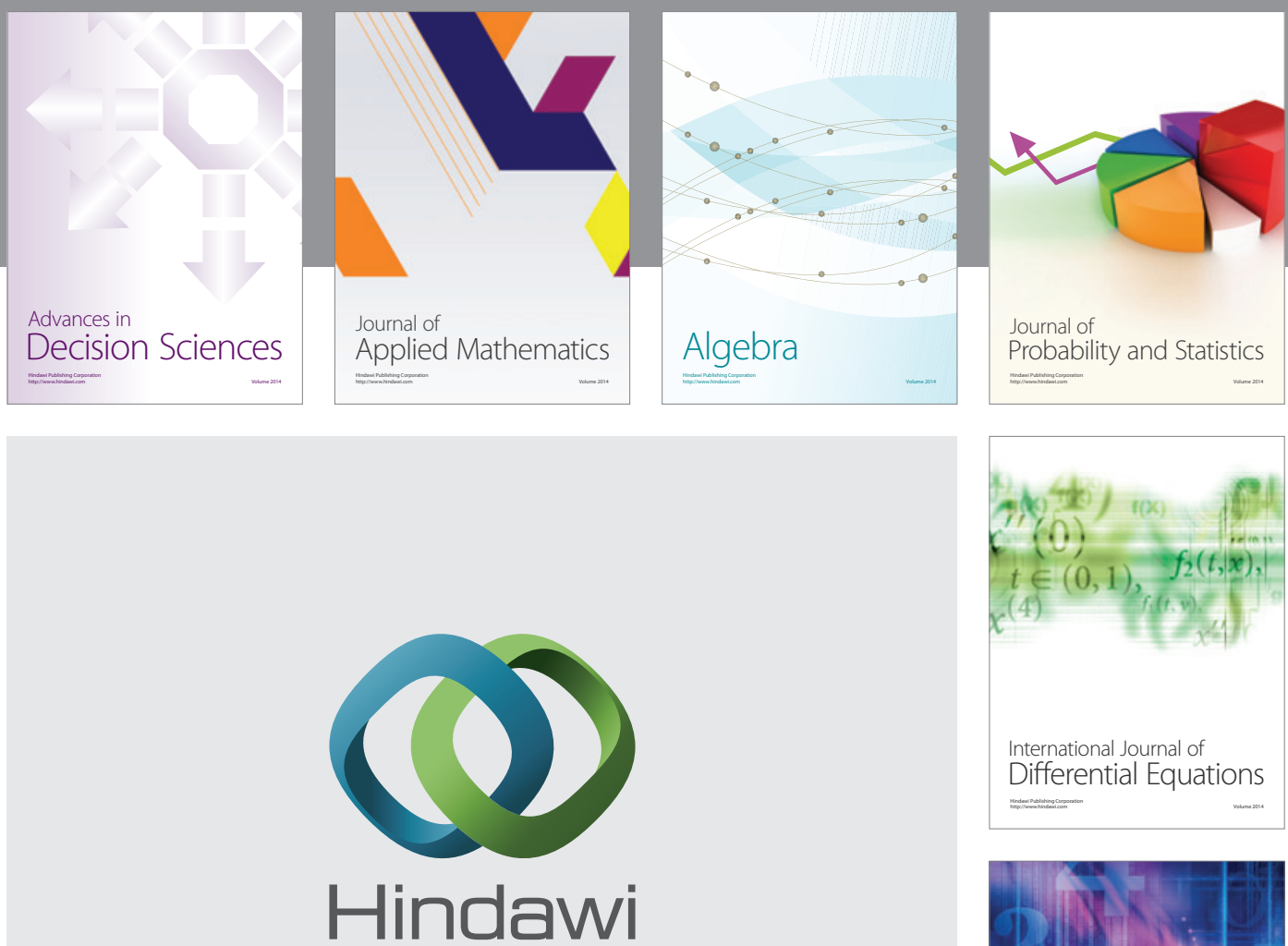

Submit your manuscripts at http://www.hindawi.com
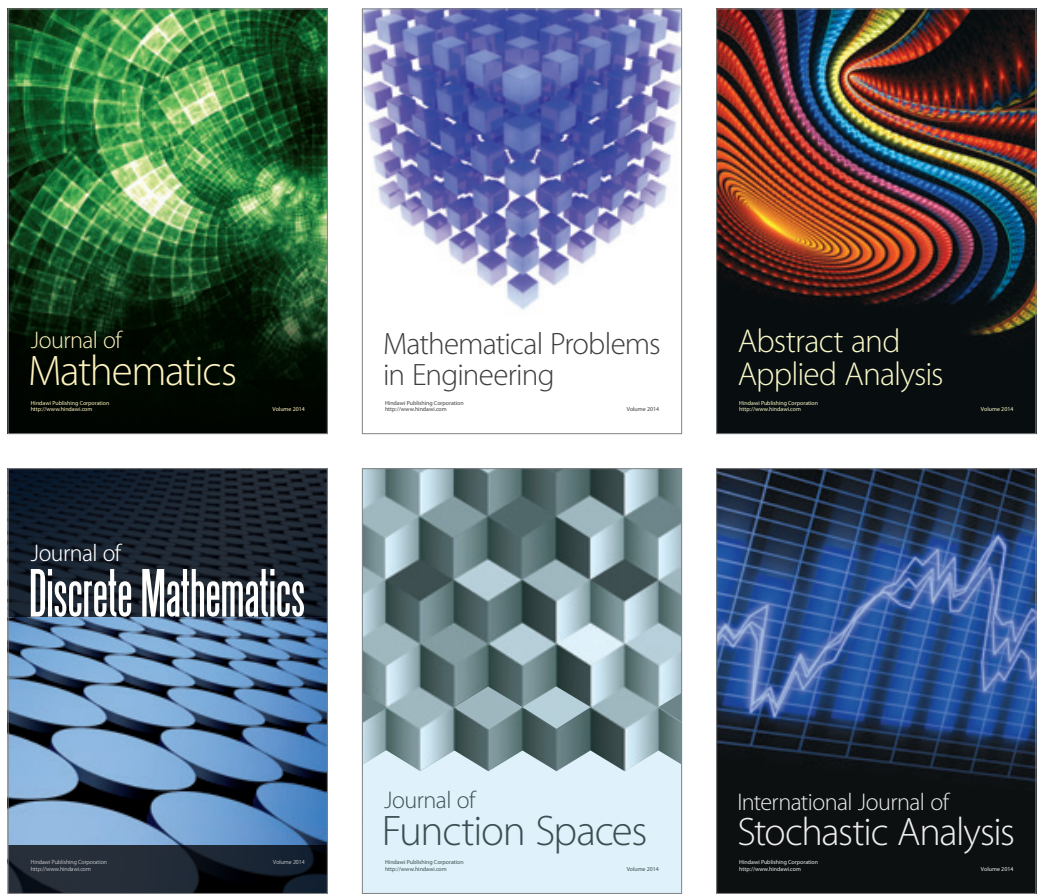

Journal of

Function Spaces

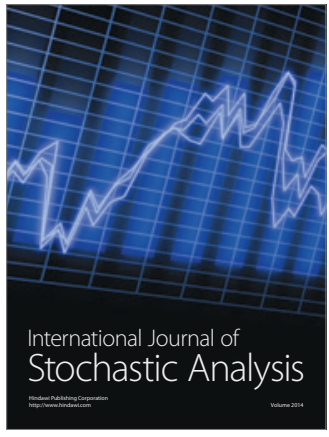

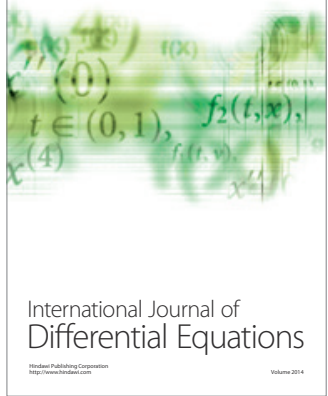
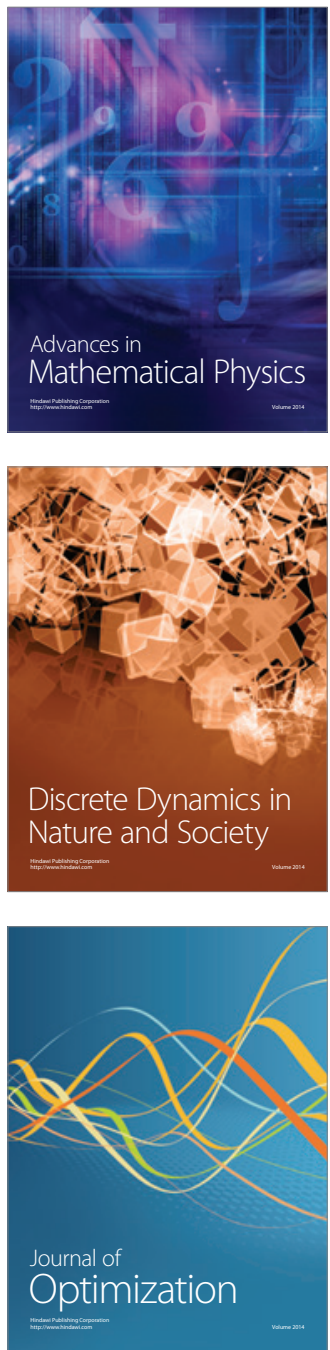